Reading a paper on "The Training of Physiologists", Prof. D. H. Smyth (University of Sheffield), who has had a lifelong interest in educational problems, began by speaking of the two different types of individual who might take up physiology as a career. One is the mathematically-minded person who, perhaps, shows his true bent in his hobby of taking the family car to pieces and putting it together again. The other is the biologically-minded individual whose leisure time has been for years expended in early nature studies and who later becomes the serious birdwatcher, fisher or amateur botanist, for he is essentially an outdoor type. While the biologically-minded type is better equipped to see the real physiological problem and has much more highly developed powers of observation, it is the mathematically-minded type who would in the end make a satisfactory analysis of the problem and offer an adequate mathematical solution. In direct contrast to Mr. Ramage, Prof. simyth was inclined to question the value of biology as a subject in the school curriculum. It is unfortunate that the educationists are as a rule inclined to put their bright pupils into physics and chemistry and the less bright into biology. What is needed is a combination of a good mathematical training and a driving interest in living organisms and how they function. Without the essential motive the more mechanically minded may become just purveyors of gadgets.

In the general discussion that followed these short papers, a questioner was assured that up to the present no difficulty has been experienced in placing women graduates in physiology, and also that no discrimination against women with first-class honours degrees exists in industry.

Dr. Bogue stressed the fact that recently graduated candidates would only be accepted for research by Imperial Chemical Industries, Ltd., if they had first-class honours degrees. $\mathrm{He}$ made it clear, however, that many second-class honours graduates were used in the more routine technical work. In his experience industry had found it well worth while to give a first-class honours chemistry graduate as much as three years special training in physiology or pharmacology to get the individual they wished.

There appears to be little accurate knowledge available of the actual number of posts open to physio- logists in Britain. In view, however, of the number of unfilled vacancies there seemed to be no likelihood of over-crowding within the next few years. Dr. Gray spoke of even third-class honours graduates having no difficulty in finding suitable work.

Prof. Gerard then spoke of his own interest in this question and in the survey made recently by a committee in the United States, and published under the title "Mirror to Physiology". He discussed the status of physiologists in America and the need for more physiologists there, and also for an improvement in the standards of the lower levels of physiology teaching.

Dr. R. H. Noltie directed attention to the development of physiology teaching in university and college departments of biology and zoology, in isolation from the traditional physiology departments.

The discussion closed with some telling remarks by Sir Charles Lovatt Evans. He reminded the meeting of the difference in rate of development which often means that the good second-class honours man eventually became the first-class researcher who makes the real advances in our knowledge. It was not always true that the first-class honours man became the first-class research worker. Sir Charles thought also that the present concern in many circles for technology and technicians is not without its dangers. Science is a way of thinking and technology is a way of doing things. In his view, there can be no doubt which is the more important.

Thus several points emerged which merit consideration at a future date : (1) The value of the teaching of biology in schools, including some elementary physiology, to all those who will not proceed later to university ; (2) the place of biology beside chemistry and physics in the school programme for those who will eventually go to a university ; (3) the integration of the teaching of physiology in colleges and universities where this is now being done outside the departments of physiology ; (4) the need for a survey of the number of physiology graduates required each year not only in the traditional physiology college and university departments but also throughout the whole field, so that training may be geared to the numbers required and diversified to meet the various new demands.
H. S. D. Garven

\title{
SEMICONDUCTOR RESEARCH
}

$I^{N}$ the ten years that have elapsed since the invention of the transistor, the close interaction between academic interest and industrial requirements in the semiconductor field has led to the growth of many industrial and government laboratories with interests in this subject. Because of the connexion with academic work, these laboratories were readily able to grow by attracting graduates of suitable calibre. This has naturally led to much research activity in the semiconductor field, and this is nowhere moro pronounced than in the United States. There the transistor was invented, and there alone has it been marketed competitively. It was therefore appropriate that the bi-annual international conference on semiconductors should at last be convened in the United States, having previously met in Britain, Holland and Western Germany. The attractive and spacious campus of the University of Rochester was selected, and the meeting was held during August 18-22 under the chairmanship of John Bardeen. Almost all centres of semiconductor research were represented.

Even with the restrictions placed by the organizers on the number of papers read at the conference, their detailed discussion is not possible here. The general headings have not changed very much since the last meeting in $1956^{1}$, but some changes of emphasis have occurred in the interim and will now be surveyed.

Excitons. There was considerable interest in excitons at the 1956 meeting, with particular reference to the pioneer work of E. F. Gross (Leningrad) and S. Nikitine (Strasbourg), who had noted exciton spectra. Their experiments, which were still questioned in 1956, have now been largely accepted, 
and they both reported at Rochester on their work. In particular, Gross showed how the quadratic diamagnetic Zeeman effect, rather than the linear paramagnetic effect, was observed in the cuprous oxide exciton spectrum. This suggests that the excitons behave here like positronium atoms, with equal effective masses for electron and hole.

The propagation of photoconductivity in single crystals of cadmium sulphide over distances of millimetres had been attributed tentatively to the properties of excitons ${ }^{1}$. This surprising result was reported in 1956 in a paper by Balkanski and Broser : the latter author now favours a different explanation of the results, namely, the scattering and re-absorption of luminescent radiation. $\mathrm{He}$ described experiments which furnish good evidence for his present view. In other papers ( $T$. Muto, Tokyo ; B. Goodman, Missouri ; W. Martienssen, Göttingen) the role of excitons in ionic crystals was also discussed.

Evidence for the creation of excitons in germanium and silicon has recently been obtained from the fine structure of the absorption edge. British work by G. G. Macfarlane and his colleagues at the Royal Radar Establishment, Malvern, as well as the theoretical interpretation by R. J. Elliott (Oxford) was given due prominence at the meeting. In the latter contribution, the new material included a discussion of the effect of the Coulomb interaction, previously neglected. Work at the Lincoln Laboratory (B. Lax et al.) has confirmed some of these results, and an exciton life-time of the order of $10^{-11}$ sec. has been suggested for germanium in zero field. Fine structure in the intrinsic recombination radiation (J. R. Haynes et al., at the Bell Telephone Laboratories) is consistent with this optical absorption work. There is, therefore, now firm experimental evidence for the existence of excitons in both elemental and compound semiconductors. It is hoped to learn more from these experiments about the exciton spectra, and about the phonons which can take part in their excitation.

Optical and magneto-optical effects. There has also been considerable progress in this field since 1956 . In a magnetic field the electron states in a band are displaced on an energy scale to give rise to the so-called Landau levels. Since the optical transitions into the conduction band depend on the density of final states, one would therefore expect oscillations if the absorption coefficient is plotted against photon energy. Such oscillations due to interband transitions have been found, notably in germanium, InSb and InAs (E. Burstein et al., Philadelphia; B. Lax, Lincoln Laboratories), and progress has been made in the interpretation of the large amount of information now available.

Low-temperature optical absorption experiments, made with the view of finding evidence for the excitation and ionization of impurities, have been pursued by H. Y. Fan et al. (Purdue), at liquid-helium temperatures and out to $300 \mu$, on various concentrations of impurities in silicon and germanium. A significant development here is that in recent years the simple hydrogen-like model for the impurities has been replaced in the interpretation of experiments of this kind by the more refined model of W. Kohn. Upon applying a magnetic field the Zeeman splitting of the excited states can be observed. In addition to the magneto-optical interband oscillations men. tioned above, a similar effect must be expected if the electrons are initially in impurity states, and this has also been observed (R. F. Wallis, Washington; W. S. Boyle, Bell Telephone Laboratories).

Reviews of work on certain infra-red phosphors (G. F. J. Garlick, Hull) and of conductivity and luminescence of diamonds (E. W. J. Mitchell, Reading) were also given.

Recombination. So far as the theory is concerned, recent efforts in this field have been devoted to the investigation of more and more mechanisms with the view of explaining the experimental results, which are still poorly understood as regards absolute values, though the situation is better if one restricts attention merely to the explanation of shapes of curves. At the meeting, reasons for the large capture crosssections $\left(\sim 10^{-14} \mathrm{~cm} .{ }^{2}\right)$ of traps in germanium and silicon were put forward (M. Lax, Bell Telephone Laboratories), and the part played by the Auger process in recombination was discussed. The main weight of research, however, has been concentrated on accumulating more experimental information. The work on the effect of dislocations on recombination-rates should here be mentioned as an active field (A. F. Gibson, Royal Radar Establishment, Malvern; P. Baruch, Paris ; R. K. Müller, Minneapolis ; H. Kawamura, Tokyo). In particular, surprisingly detailed structure was reported in the recombination radiation through dislocation in germanium at $20^{\circ} \mathrm{K}$. (C. Benoit à la Guillaume, Paris).

Perhaps the most interesting 'hard' results (because they are older and confirmed) to be discussed were those on deep-level impurities in germanium, where different experimental methods led to the same picture, namely, the possibility of multiple ionization of the impurities (W.W. Tyler, Schenectady). Though the statistics of recombination through such centres appears to bo well understood, a satisfactory calculation of the position of the energy-levels is still outstanding.

Hot electrons, high-field effect and impact ionization. The term 'hot electrons' is due to Shockley (1951). (It is perhaps not generally known that the concept of effective electron temperatures in solid state physics appears to have been used first by $\mathrm{H}$. Fröhlich ${ }^{2}$.) The three concepts, hot electrons, high-field effect and impact ionization, are closely related, since a high electric field causes electron-hole pairs to be created by impact ionization-a conduction electron knocks an electron out of the valence band-and it also causes electrons to gain more energy than they can lose to the lattice, so that they can attain a temperature greater than that of the lattice. Among the main advances made since 1956 is the now widespread appreciation that one can realize, by impact ionization, quantum efficiencies in excess of unity. This is largely due to the work on the inner photoelectric effect by the Czech school (J. Tauc, Prague).

Another interesting effect is the evidence of anisotropy in electric conduction at high fields, which can have the effect that some valleys (of the energy surfaces in $k$-space) become hotter than others, with a consequent transfer of electrons from the hotter to the colder valleys (W. Sazaki et al., Tokyo). Attention was also directed to the importance of electron electron scattering with the resultant emission of optical phonons as a loss mechanism for hot electrons (J. B. Gunn, Vancouver). Interesting results, recently published in Physical Review Letters, on the use of pulsed current-voltage measurements to show the incidence of impact ionization in $n$-type single crystals of InSb were also presented (M. C. Steele and M. Glicksman, Princeton). 
Other transport effects. In this field a large amount of detail has been filled in, and some was reported at the meeting, on both the older and the newer semiconductors. The main Russian contribution may be mentioned here, namely, to direct attention again to the low-mobility semiconductors (A. Joffé, Leningrad), which may raise fundamental problems. For example, if the mean free path of electrons is of the order of : $l=10^{-8} \sqrt{ }\left(m_{e} / m\right) v \mathrm{~cm}$., where $v$ is the mobility and $m_{e}$ the effective mass of electrons, then $l$ is less than atomic distances for $v<10 \mathrm{~cm} .{ }^{2} / \mathrm{volt}$ sec., and therefore ceases to have a meaning. The concept of energy-bands itself may become inapplicable. Impurity scattering and mobility theory would both require extensions for this class of substances. The view was also expressed that the change of electrical properties during melting suggests that the zone structure of solids depends only on short-range order. Great difficulties, however, stand in the way of understanding disordered structures, to which a paper by C. Domb et al. (London) was devoted.

Substances which exhibit impurity-band conduction present some features of a disordered low-mobility system, but little progress in the understanding of this phenomenon appears to have been made, though interesting papers on this topic were presented at the meeting (germanium, H. Fritsche, Chicago ; silicon, T. Longo et al., Purdue; InSb, R. J. Sladek, Pittsburgh).

Low-temperature photoconductivity in silver halides was described by J. W. Mitchell (Bristol) and F. C. Brown (Urbana).

Bonds. In connexion with the renewed attempt to look beyond the band picture, a number of substances have been studied recently the properties of which may possibly be more readily understood in terms of some chemical-bond picture. A curiosity among these is the compound AuCs, with two elements of the first group, which is a large-gap $\left(E_{g} \simeq 2.9 \mathrm{\Theta V}\right.$.) semiconductor (W. E. Spicer and A. H. Sommer, Princeton). Also of interest are the $A^{\text {III }} B^{\text {VI }}$ compounds from the third and sixth group (for example, $\mathrm{GaTe}$ ), which have been investigated by $\mathrm{E}$. Mooser et al. (Ottawa). These are semiconductors although they have nine valence electrons per anion, that is, one more than is normal in such semiconductors.

Bands. There was at the 1956 meeting an almost complete absence of energy-band calculations ${ }^{1}$, but this was not true of the 1958 meeting. Several American workers have recently reconsidered the status of the one-electron approximation, and W. Kohn (Pittsburgh) spoke on the justification of the one-electron effective-mass approximation from the many-electron Schrödinger equation. The effect of a magnetic field may require further consideration here. The use of potential operators in band calculations was also discussed. Particularly the k . p approach (E. O. Kane, Schenectady) has in recent years proved very useful, though it was suggested by J. C. Philips (Bell Telephone Laboratories) that rather large corrections may be required. Band forms discussed at the meeting included $\mathrm{ZnS}(\mathrm{J}$. L. Birman, Bayside), InAs (F. Stern, Silver Springs), GaAs (R. Braunstein, Princeton), apart from silicon and germanium.

Surfaces. There has been steady consolidation in this field, which was surveyed by A. Many (Jerusalem). The distinction between fast and slow states appears to remain useful, and the origin of the fast states is still not understood, though they are believed to reside in the transition region from the bulk material to the surface oxide. The time-lag which usually exists before experiments on the bulk material are attempted for a restricted part of the surface layer is, however, shortening, and optical, magnetic and transport properties of surfaces were discussed (for example, by J. N. Zemel and R. L. Petritz, Silver Springs). The adsorption on germanium and silicon appears to follow a logarithmic rate law (S. Wolsky, Waltham), familiar in oxidation and chemisorption studies. Reproducible 'clean' ('Farnworth') surfaces can be produced by ion-bombardment or heating techniques, and these have now become standard procedures.

The meeting closed with a very useful summary session, in which L. Apker, W. H. Brattain, H. Brooks, C. Herring and D. Polder took part, and one may express the hope that this will become an increasingly important pattern of future meetings. The proceedings will be published early in 1959 in the Journal of the Physics and Chemistry of Solids, and the next meeting will probably take place in Prague in 1960.

P. T. LANDSBERG

Nature, 178, 1156 (1956).

2 Proc. Roy. Soc., A, 188, 521 (1947).

\section{THE EUROPEAN NUCLEAR ENERGY AGENCY}

$\mathrm{U}^{\mathrm{N}}$ NDER the title "The European Nuclear Energy Agency and the Eurochemic Company*, the Organization for European Economic Co-operation has published the first report of its Steering Committee for Nuclear Energy. It includes the Statute for the European Nuclear Agency, which the Committee recommends the Council should adopt, as well as two draft conventions, one on the establishment of security control in the field of nuclear energy and the other on the constitution of the European Com-

* Organisation for European Economic Co-operation. European Nuclear Energy Agency and the Eurochemic Company. First Report Pp. 192. (Paris: Organisation for European Economic Co-operation, 1958.) 750 French francs; $128 . ; 2$ dollars. pany for the Chemical Processing of Irradiated Fuels (Eurochemic). The former is to be recommended to member governments for signature and the latter is to be submitted for signature to the governments interested. Amendments to its Statute are also proposed by the Study Group for the Chemical Processing of Irradiated Fuels and authorization is requested for expenditure by the Study Group of 500,000 European Payments Union units of account. The Study Group also recommends that the proposed plant should be located at $\mathrm{Mol}$ in Belgium, but if it were decided later to construct a large plant the most suitable site is considered to be that proposed by Norway. The present plant is estimated to cost 